\title{
Advanced Characterisation of a Sensor System for Droplet- Based Microfluidics
}

This paper was downloaded from TechRxiv (https://www.techrxiv.org).

\section{LICENSE}

CC BY-NC-SA 4.0

SUBMISSION DATE / POSTED DATE

$11-01-2021 / 13-01-2021$

\section{CITATION}

Bartunik, Max; Fleischer, Marco; Haselmayr, Werner; Kirchner, Jens (2021): Advanced Characterisation of a Sensor System for Droplet-Based Microfluidics. TechRxiv. Preprint. https://doi.org/10.36227/techrxiv.13554479.v1

$\mathrm{DOI}$

10.36227/techrxiv.13554479.v1 


\title{
Advanced Characterisation of a Sensor System for Droplet-Based Microfluidics
}

\author{
Max Bartunik*, Marco Fleischer*, Werner Haselmayr ${ }^{\dagger}$ and Jens Kirchner* \\ ${ }^{*}$ Institute for Electronics Engineering, Friedrich-Alexander-Universität Erlangen-Nürnberg (FAU), Erlangen, Germany \\ max.bartunik@fau.de \\ ${ }^{\dagger}$ Institute for Communications Engineering and RF-Systems, Johannes Kepler University Linz (JKU), Linz, Austria
}

\begin{abstract}
Droplet-based microfluidics show a large potential for lab-on-chip applications and new data transmission scenarios. Microfluidic chips contain channels in the submillimeter range allowing for flow of droplets. In a previous contribution, a new sensor design for droplet size and colour detection, consisting of an infrared and a colour sensor, was presented and a first proof-of-concept was shown. In this work, an in-depth analysis of both concepts is presented. In particular, we show that a high precision can be achieved when using the sensor to measure droplet sizes while using video processing software as reference. Furthermore, a colour alphabet consisting of 126 individual values is transmitted and detected using a machine learning model. The high specificity of achieved colour measurement allows both for colour coded data transmission scenarios and the analysis of colour reagents in lab-on-chip applications.

Index Terms-Droplet detection, microfluidic environment, colour coding, optical sensor
\end{abstract}

\section{INTRODUCTION}

Droplet-based microfluidic setups provide a promising platform for various lab-on-chip (LoC) applications, such as drug screening or capillary electrophoresis [1], [2]. In some cases it is desirable to encode and transmit information using droplets or measure droplet parameters in such a setup [3]-[5]. Dropletbased communication was presented as a theoretical principle in [6], a demonstration of first practical applications was performed in [7].

A sample use-case for data transmission in LoC applications can be found in [4], where a system for LoC networking is proposed. In this case, information is encoded as distance between droplets to achieve dynamic droplet routing in the network. Moreover, in [8] an LoC application for droplet detection and characterisation, to perform lab tests on cancer cells, is presented.

To facilitate the broad spectrum of applications, which require a reliable droplet parameter detection, a cost-efficient and portable device is desirable. Although various methods for droplet detection, by measuring capacitance in a microfluidic channel, fluorescence of droplets or analysing video feedback, have been presented (e.g. [8]-[15]), they require either a complex lab setup or rely on computationally expensive video processing. A state-of-the-art video processing tool was presented in [16], which enables the evaluation of multiple droplet parameters, such as size and velocity. Colour coded characterisation of droplets was achieved in [17] by injecting droplets with coloured polystyrene beads. These were detected under a bright-field microscope with the use of machine learning to differentiate between 20 different states.

A promising low-cost optical sensor device with a compact design, suitable for detection of various droplet parameters, such as size and colour in a microfluidic setup, was proposed in [5]. The aim of this work is to further investigate the capabilities of this sensor device for droplet characterisation in terms of droplet size and colour, which can be used for information encoding in data transmission scenarios, as well as for other LoC applications. In contrast to [17], we propose a more flexible coding system for colour coded data transmission, using ink to produce coloured droplets, allowing for the creation of a large coding alphabet by mixing three fundamental colours.

In the following, we will first outline the used testbed and especially the specifications of the sensor device. In Section III the performed experiments and results to determine the sensors capabilities regarding both measurement of droplet size and colour are presented. We conclude the article with a discussion of the results and an outlook on future work.

\section{TESTBED}

The testbed setup consists of a microfluidic chip with a Tjunction, two pressure driven pumps and the droplet detection device. Inside the microfluidic chip the transmission channel is supplied with a continuous phase of oil by one of the pumps. At the T-junction, coloured ink is injected with the second pump to create a droplet that travels with the continuous phase through the transmission channel to the detection device.

The droplet detection device was custom made at the Institute for Electronics Engineering of the FAU, Germany. It consists of a printed circuit board equipped with an infrared sensor (VBPW34FAS, Vishay), a six channel spectral sensor (AS7262, ams AG) and a microprocessor. To facilitate microfluidic droplet detection a housing was constructed so that the microfluidic channel can be positioned precisely above the two sensors. Two LEDs (infrared and white) are used as light sources for the sensors. The printed circuit board with the sensor components is shown in Figure 1.

The infrared sensor is sensitive to light with a wavelength of $940 \mathrm{~nm}$, which shows a high absorption in water, and is used for droplet presence/absence detection. The spectral sensor (AS7262, AMS AG) has six individual photo sensors covering a range from $430 \mathrm{~nm}$ to $670 \mathrm{~nm}$. It can primarily be used 


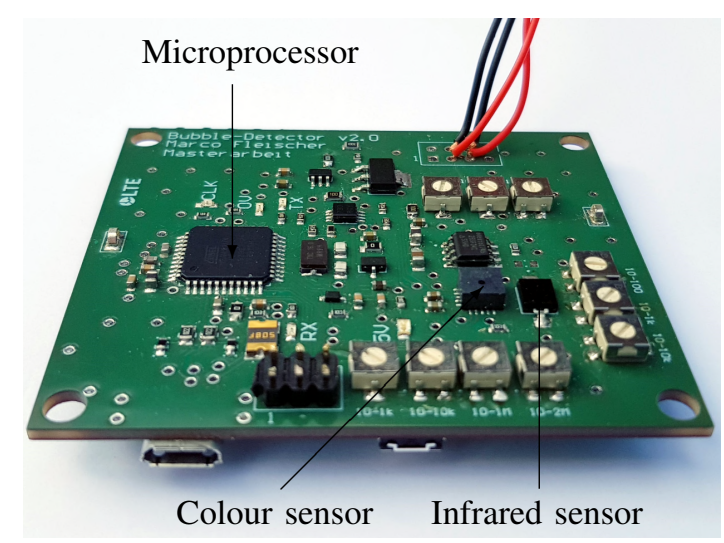

Fig. 1. Microfluidic droplet detection device consisting of an infrared and a colour sensor. Data is recorded with a microprocessor and can be retrieved over a serial connection. The attached cables connect to the separate LED light sources [5].

to determine the colour of a detected droplet. Both sensors in parallel may be used to measure droplet velocity in the microfluidic channel and size.

\section{RESUltS}

Two different droplet characteristics were investigated using the developed sensor device. First, the droplet size was measured and compared to the results of video processing software. Second, the sensitivity to changes in droplet colour was determined. This is essential to colour coded data transmission scenarios and detection of a coloured reagent in LoC setups.

\section{A. Measurement of Droplet Size}

To determine the droplet size, both the infrared and the spectral sensor are used. Due to the distance between the two sensors placed along the transmission channel, the droplet velocity $v_{\text {drop }}$ and length $l_{\text {drop }}$ can be calculated according to

$$
l_{\text {drop }}=v_{\text {drop }} t_{\text {drop }}=\frac{\Delta d}{\Delta t} t_{\text {drop }},
$$

where $t_{\text {drop }}$ denotes the observed droplet duration at the sensor, $\Delta d=6.35 \mathrm{~mm}$ the fixed distance between the two sensors and $\Delta t$ the delay between detection of the same droplets rising edge at the two sensors.

We demonstrated the basic principle for droplet size detection in [5]. Now, to mitigate a linear offset between the calculated droplet size using the developed sensor and the result of a camera-based video processing software used as reference, we extend (1) with correction factors $m$ and $c$ :

$$
l_{\text {drop }}=m \frac{\Delta d}{\Delta t} t_{\text {drop }}+c
$$

For video processing we used the software "Droplet Morphometry and Velocimetry" (DMV) from [16], which allows the determination of various droplet parameters like position, velocity and size, by evaluating a provided video file. With the reference values, measured with the DMV software, we can improve and provide an in-depth analysis of the sensors performance.

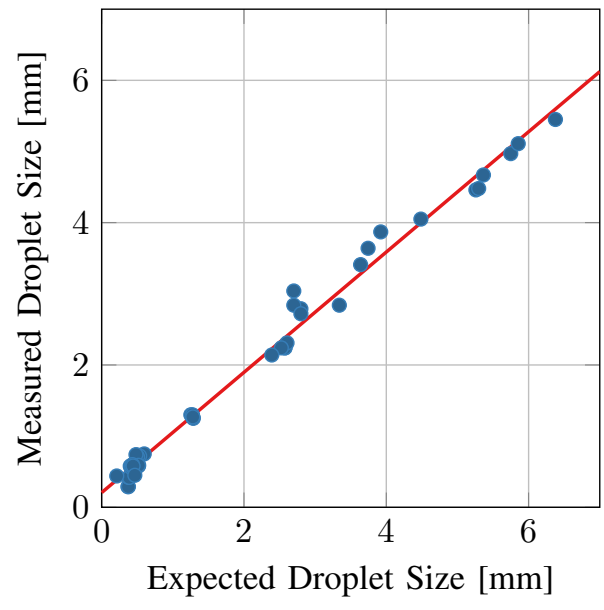

Fig. 2. Measured droplet sizes for a training set of 45 droplets with DMV software as reference (expected droplet size). In some cases multiple measurement points overlap. The fitted linear relation is shown in red.

To estimate the correction factors $m$ and $c$ in (2) we measured the sizes of a droplet sample set with 45 droplets in the range of $0.21 \mathrm{~mm}$ to $6.37 \mathrm{~mm}$, with the video processing software and the sensor device. A linear relation was then fitted using this training set and the correction factors determined as $m=0.8452$ and $c=0.2061 \mathrm{~mm}$.

Figure 2 shows the training set as the measured droplet size according to (1) in relation to the actual droplet size determined using video processing software. Furthermore, the fitted linear relation is shown.

To evaluate the droplet size precision a second data set of 70 droplets was recorded. The measurement results can be seen in Figure 3 as deviation from the expected value. The error only exceeds $0.5 \mathrm{~mm}$ in three cases and is independent of droplet size.

\section{B. Measurement of Droplet Colour}

Using the spectral sensor the colour of microfluidic droplets can be determined. Again, a proof of concept with six different colours was made in [5]. We now present a detailed analysis of the sensor specificity and inspect a sample transmission scenario with an alphabet of 126 colours.

Each droplet colour of the transmission alphabet consists of a different mixture of ink made up of the three fundamental colour components cyan, magenta and yellow. The used mixtures to make up the alphabet can be seen in Figure 4.

The six spectral values for each droplet are recorded using the colour sensor. To estimate the transmitted colour value machine learning, using a linear regression algorithm for each of the three fundamental colour components, was employed with a training set of 252 sample values (two samples for each alphabet value). Unfortunately, a larger training set was not possible due to the high workload connected to generating the coloured droplets. In future work, this could be automated by mixing the three fundamental colour components on-chip. 


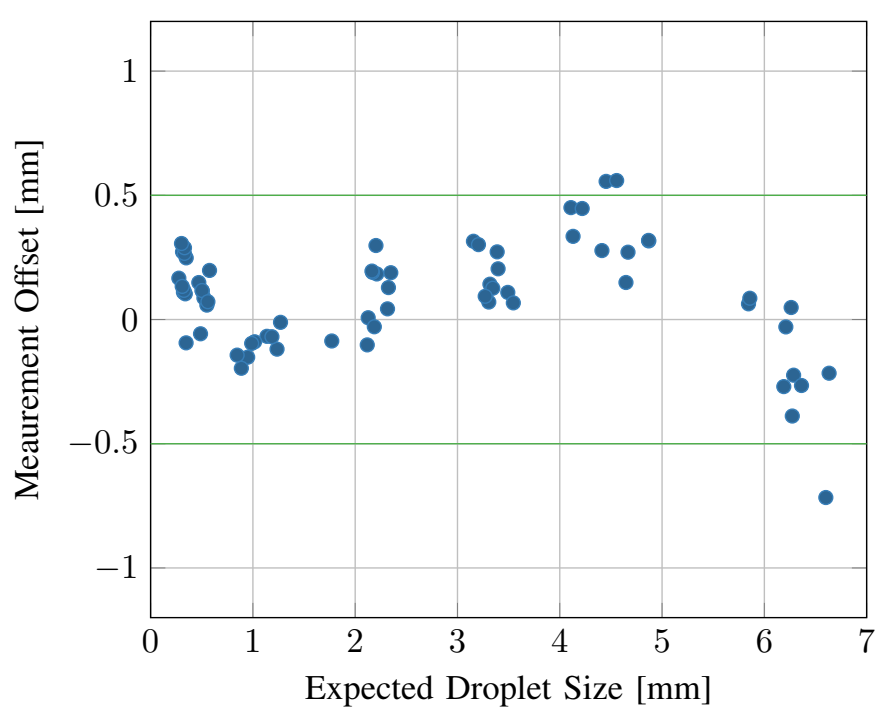

Fig. 3. Measured droplet size error for 70 droplets according to (2) using the DMV software as reference (expected droplet size). The maximal offset is $0.716 \mathrm{~mm}$, while most errors are smaller than $0.5 \mathrm{~mm}$.
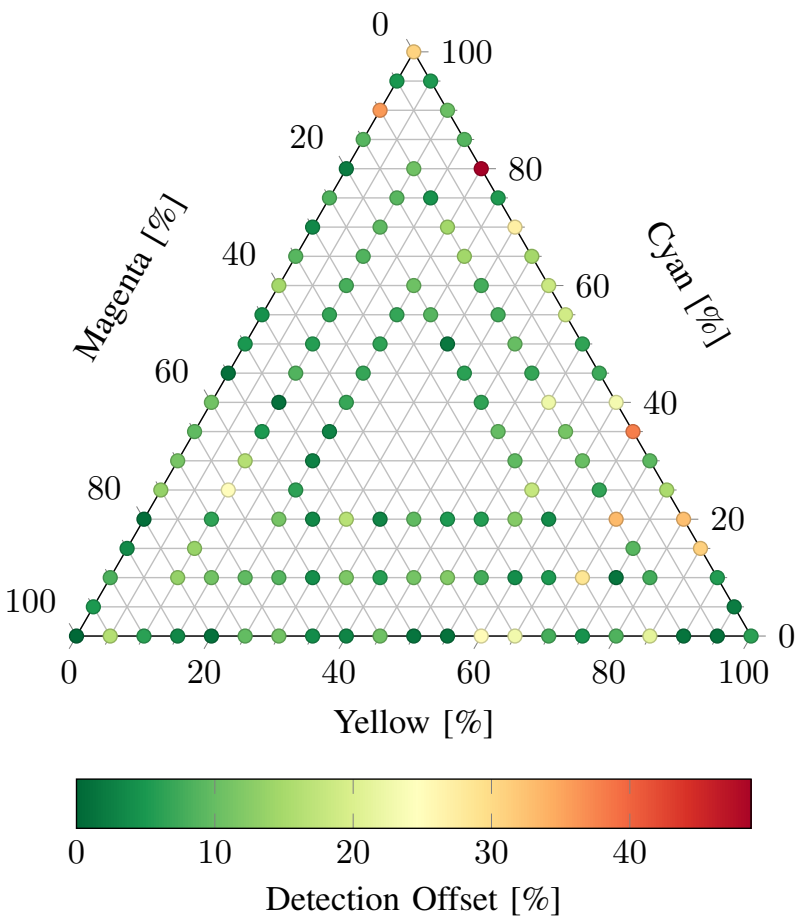

Fig. 4. Prediction error using machine learning for a transmitted colour alphabet with 126 different values. One droplet was recorded for each colour value. The position in the coordinate system of the fundamental colours cyan, magenta and yellow corresponds to the transmitted colour value, the detection offset for each value is colour coded. The average detection offset is $10.1 \%$.
As we observe the average result of the whole alphabet, the measured values provide a sufficient data set.

A colour sample was created by mixing $1 \mathrm{ml}$ of ink with $9 \mathrm{ml}$ of water. The various colour values of the provided alphabet, consisting of up to three colour components each, were gained by setting one colour component to a fixed concentration and varying the other two components in $5 \%$ steps. Finally, we determined the prediction accuracy using a further data set and applying the previously trained machine learning model.

Figure 4 shows the resulting detection precision for the individual values of the colour alphabet. The position of the data point corresponds to the transmitted colour mixture, the colouring shows the distance between transmitted and detected colour. The average offset is $10.1 \%$. In $85.7 \%$ of the observed cases the detection offset is smaller than $17 \%$. With an increased training set size, these values may be further improved in the future.

\section{CONCLUSiON}

We thoroughly investigated the presented sensor device regarding droplet size and colour detection. In most cases the size can be determined to an accuracy of $0.5 \mathrm{~mm}$, allowing for transmission scenarios where information is encoded as droplet length. Furthermore, the ability for size determination can be used as a control mechanism for droplet-on-demand generation. In contrast to the DMV software, that was used as reference, the size measurements can be performed in realtime without significant hardware, whereas video processing is computationally more expensive and requires a camera setup in an environment with controlled lighting.

Colour coding presents a further possibility to transmit data with ink droplets in a microfluidic setup. As we could demonstrate with a sample alphabet of 126 colours, colour coded data transmission with machine-learning-based detection is feasible with the provided sensor device. This allows for a significant increase of achievable information rate in comparison to previous approaches with presence/absence or distance encoding. In contrast to [17] no beads have to be introduced to achieve colour coding. Furthermore, a large colour alphabet made up of three fundamental colours can be used, wheras in [17] only 20 different predetermined bead colours were differentiated. In addition, the device could be used for the analysis of colour reagents (such as a $\mathrm{pH}$-value) in LoC application.

In future work we intend to demonstrate both transmission scenarios with colour coded information and the application of the sensor device in LoC setups. Furthermore, the provided colour alphabet and sensor prediction result can be improved with a significantly larger training set containing a fine grid of the complete colour spectrum. Finally, precision of droplet generation in the testbed setup may be improved by applying the measured droplet size in a feedback loop.

\section{ACKNOWLEDGMENT}

The authors would like to express their sincere gratitude to Dominik Lehner for his support with the microfluidic setup. 


\section{REFERENCES}

[1] W. Haselmayr, M. Hamidovic, A. Grimmer, and R. Wille, "Fast and flexible drug screening using a pure hydrodynamic droplet control," in Proc. of the $5^{\text {th }}$ Eur. Conf. on Microfluidics, Mar. 2018, pp. 1-4.

[2] D. Mark, S. Haeberle, G. Roth, F. von Stetten, and R. Zengerle, "Microfluidic lab-on-a-chip platforms: requirements, characteristics and applications," Chemical Society Reviews, vol. 39, p. 1153-1182, Jan. 2010.

[3] A. Biral, D. Zordan, and A. Zanella, "Transmitting information with microfluidic systems," 2015 IEEE International Conference on Coтmunications (ICC), pp. 1103-1108, Jun. 2015.

[4] E. D. Leo, L. Galluccio, A. Lombardo, and G. Morabito, "Networked labs-on-a-chip (nloc): Introducing networking technologies in microfluidic systems," Nano Communication Networks, vol. 3, no. 4, pp. 217 228, Dec. 2012.

[5] M. Bartunik, M. Fleischer, W. Haselmayr, and J. Kirchner, "Colourspecific microfluidic droplet detection for molecular communication," arXiv:2006.09774, 2020, to be published in ACM Int. Conf. Nanoscale Comput. Commun. - NANOCOM '20.

[6] E. De Leo, L. Donvito, L. Galluccio, A. Lombardo, G. Morabito, and L. M. Zanoli, "Communications and switching in microfluidic systems: Pure hydrodynamic control for networking labs-on-a-chip," IEEE Trans. Commun., vol. 61, no. 11, pp. 4663-4677, Nov. 2013.

[7] M. Hamidović, U. Marta, G. Fink, R. Wille, A. Springer, and W. Haselmayr, "Information encoding in droplet-based microfluidic systems: First practical study," in Proc. Sixth Annu. ACM Int. Conf. Nanoscale Comput. Commun. - NANOCOM '19, no. 26. Dublin: Association for Computing Machinery, Sep. 2019, pp. 1-6.

[8] A. H. Tkaczyk, E. R. Tkaczyk, T. B. Norris, and S. Takayama, "Microfluidic droplet consistency monitoring and cell detection via laser excitation," J. Mechanics Medicine Biology, vol. 11, no. 1, pp. 1-14, Mar. 2011.

[9] B. de Heij, B. van der Schoot, H. Bo, J. Hess, and N. F. de Rooij, "Characterisation of a fl droplet generator for inhalation drug therapy," Sensors and Actuators A: Physical, vol. 85, no. 1-3, pp. 430-434, Aug. 2000.

[10] M. Hamidović, U. Marta, H. Bridle, D. Hamidović, G. Fink, R. Wille, A. Springer, and W. Haselmayr, "Off-chip-controlled droplet-on-demand method for precise sample handling," ACS Omega, vol. 5, no. 17, pp. 9684-9689, Apr. 2020.

[11] A. Golberg, G. Linshiz, I. Kravets, N. Stawski, N. J. Hillson, M. L. Yarmush, R. S. Marks, and T. Konry, "Cloud-enabled microscopy and droplet microfluidic platform for specific detection of escherichia coli in water," PLoS ONE, vol. 9, no. 1, pp. 1-9, Jan. 2014.

[12] B. Chen, X. Han, Z. Su, and Q. Liu), "Fluorescence detection system for microfluidic droplets," in Sixth AIP Int. Conf. Comput.-Aided Design, Manuf., Model. Simul. (CDMMS 2018), vol. 1967. Nanjing: AIP Publishing, May 2018, pp. 1-5.

[13] X. Niu, M. Zhang, S. Peng, W. Wen, and P. Sheng, "Real-time detection, control, and sorting of microfluidic droplets," Biomicrofluidics, vol. 1, no. 4, pp. 1-12, Sep. 2007.

[14] C. Elbuken, T. Glawdel, D. Chan, and C. L. Ren, "Detection of microdroplet size and speed using capacitive sensors," Sensors and Actuators A: Physical, vol. 171, no. 2, pp. 55-62, Nov. 2011.

[15] P. K. Isgor, M. Marcali, M. Keser, and C. Elbuken, "Microfluidic droplet content detection using integrated capacitive sensors," Sensors and Actuators B: Chemical, vol. 210, pp. 669-675, Apr. 2015.

[16] A. S. Basu, "Droplet morphometry and velocimetry (dmv): a video processing software for time-resolved, label-free tracking of droplet parameters," Lab on a Chip, vol. 10, pp. 1892-1901, Mar. 2013.

[17] C.-M. Svensson, O. Shvydkiv, S. Dietrich, L. M. T. Weber, M. Choudhary, M. Tovar, M. T. Figge, and M. Roth, "Coding of experimental conditions in microfluidic droplet assays using colored beads and machine learning supported image analysis," small, vol. 15, no. 4, pp. 1-14, Dec. 2018. 